\title{
Design and Characteristic Study of a Novel Internal Cooling High Temperature Superconducting Composite Cable with REBCO for Energy Storage Applications
}

\author{
Jiahui Zhu, Panpan Chen, Hongjie Zhang, Ming Qiu, Huiming Zhang, Jun Gong, Yuanyuan He and Min Zhang
}

\begin{abstract}
High temperature superconducting magnetic energy storage systems (HTS SMES) have attracted significant attention for fast response and ensure a reliable power supply. However, the current carrying capacity of single superconducting tape often meets limitation for the large scale HTS SMES applied in the power grid. Therefore, a high temperature superconducting composite cable with inner helical cooling tunnel and $\mathrm{kA}$ class current carrying ability is proposed for SMES magnet by using REBCO tape. The critical current characteristics of this internal cooling composite cable are analyzed considering the influence of anisotropy in magnetic field. A $100 \mathrm{~m}$ length, high temperature composite cable is manufactured in China Electric Power Research Institute (CEPRI). The critical current experimental system with a $3 \mathrm{kA}$ DC current power source and a high-precision Digital Data Acquisition (DAQ) system have been set up to investigate the current carrying ability of a straight and a bending demo composite cable in LN2. The results show that critical current of HTS composite cable consisted of 4 REBCO tapes can achieve $780 \mathrm{~A}$ at $77 \mathrm{~K}$ self-field and the experimental I-V curve of each REBCO tape in the composite cable is not uniform because of the influence of anisotropy. When the HTS composite cable is bent, its critical current is about $90 \%$ of the straight HTS composite cable. Therefore, the design method and the proposed experimental system are proved to be effective as well.
\end{abstract}

Index Terms-High temperature superconducting composite cable, inner helical cooling, REBCO, SMES

\section{INTRODUCTION}

$\mathbf{H}$ igh temperature superconducting energy storage system (HT SMES) stores energy in the magnetic field produced by a persistent current in a superconducting loop. So that HT SMES has several significant advantages: a) larger power density than other energy storage system, b) more than $85 \%$ efficiency, c) a substantially faster response speed, d) infinite charge and discharge cycles [1].

There are several completed and ongoing HTS SMES projects for power system applications [2, 3]. Superpower Inc,

Manuscript is submitted on 16, August, 2017. This work was supported by the China State Grid Corporation Science and Technology Project under Grant No. DG71-16-002, DG83-17-002, DG71-17-020.

Jiahui Zhu is with the China Electric Power Research Institute, Beijing, 100192, China (e-mail: zhujiahui@epri.sgcc.com.cn).

Panpan Chen, Hongjie Zhang, Ming Qiu, Huiming Zhang are with the China Electric Power Research Institute, Beijing, 100192, China (e-mail: chenpanpan1@ epri.sgcc.com.cn).
Houston University and Brookhaven National Lab are aiming to deliver a $2.5 \mathrm{MJ}$ system for load leveling in medium voltage $15-36 \mathrm{kV}$ distribution networks. In 2013, Germany also proposed a new type of 48 GJ hybrid energy storage system LIQHYSMES to solve the problem of grid fluctuation caused by renewable energy integration [4]. Form these above application, we find the large capacity energy storage magnet usually wants the current can be as large as possible. However, the critical current of single commercial HTS tape used in SMES can't meet this requirement. And it is difficult and costly to increase the current by paralleling coils or lowering the operating temperature.

In recent years, there are some literatures describing different kinds of superconducting composite conductor, such as twisted stacked-tape cable (TSTC) [5-8]. However, they didn't have enough considerations for the power grid application, for example in SMES. In 2016, China Electric Power Research Institute (CEPRI) has firstly designed a 3 MJ HTS SMES applying a proposed twisted stacked-tape in tube (TSTT) composite cable [9].

This paper proposes a TSTT composite cable integrated by four REBCO tapes and a constant aluminum jacket. The electromagnetic characteristics and current variation considering the anisotropy of REBCO and critical current are analyzed by using COMSOL software. A $100 \mathrm{~m}$ length, TSTT composite cable is manufactured and tested in CEPRI for the first time. The calculated and measured critical current of a straight and a bent TSTT composite cable are compared. The results show that the critical current of TSTT composite cable has achieved 780 A@77K which satisfied the current performance requirement for winding a 3 MJ HT SMES.

Jun Gong is with the Beijing Jiao-tong University, Beijing, 100044, China (email:835473391@qq.com).;

Yuanyuan He is with the Anhui hongyuan special cable group co. LTD, Wuwei, 238371, China (e-mail: hy817815@126.com).

Min Zhang is with the Department of Electronic \& Electrical Engineering, University of Bath, Bath, BA2 7AY, United Kingdom(e-mail: M.Zhang2@bath.ac.uk). 


\section{InTERnAL COOLING High TeMPERATURE COMPOSITE CA- BLE DESIGN}

\section{A. Structure design}

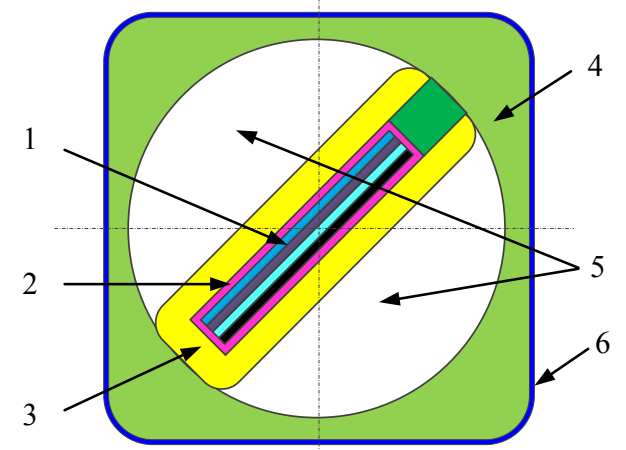

Fig. 1. sketch of inner helical cooling TSTT composite cable.

The structure schematic diagram of the composite cable is shown in Fig.1. It mainly consists of six parts: (1) superconducting core. It has 4-ply REBCO superconducting tapes stacked in parallel and twisted in a certain pitch (the total number of tapes can be increased); (2) soft metal buffer layer using silver-plated copper wire; (3) inner aluminum coating layer; (4) aluminum jacket with square outer wall and round inner wall; (5) cooling channel flowing liquid nitrogen or gas helium; (6) insulation layer of polyimide film.

This composite cable has some advantages for constructing a SMES. Firstly, the stacked tapes have been twisted so the anisotropy of HTS wires can be reduced to make current distribution more uniform, which is beneficial to enhance the critical current and reduce AC losses. Secondly, the spiral inner cooling channels can increase the contact surface to improve the cooling efficiency of magnet. Thirdly, the aluminum jacket acts as a stabilizer to withstand the fault current for SMES quench protection. Finally, the compact structure can greatly reduce the size of the high temperature energy storage magnet. The specification of a TSTT composite cable has shown in Table.1.

TABLE I

SPECIFICATION OF THE INTERNAL COOLING TWISTED STACKED-TAPE IN TUBE COMPOSITE CABLE FOR HTS SMES

\begin{tabular}{cl}
\hline \hline Parameters & \multicolumn{1}{c}{ Value } \\
\hline Sectional dimension & $13 \mathrm{~mm} \times 13 \mathrm{~mm}$ \\
Length & $100 \mathrm{~m}$ \\
Inner diameter & $10.8 \mathrm{~mm}$ \\
Pitch & $>200 \mathrm{~mm}$ \\
Superconducting core size & $9.6 \mathrm{~mm} \times 4.8 \mathrm{~mm}$ \\
Thickness of soft metal buffer layer & $0.5 \mathrm{~mm}$ \\
Critical tensile stress $(77 \mathrm{~K})$ & $250 \mathrm{MPa}$ \\
Critical tensile strain $(77 \mathrm{~K})$ & $0.3 \%$ \\
Minimum bending diameter & $300 \mathrm{~mm}$ \\
REBCO tape number & 4 \\
Critical current $(77 \mathrm{~K})$ & $700 \mathrm{~A}$ \\
Length per cable & $100 \mathrm{~m}$ \\
\hline
\end{tabular}

\section{B. Critical current characteristics analysis}

A T-A equation model is applied for the first time to calculate the magnetic field and current distribution of each REBCO tape in the composite cable considering the influence of anisotropy in magnetic field. It is a general, flexible and efficient FEM model which can be implemented in commercial FEM software to analyze complex geometries. The T-A governing equation based on Faraday's law is shown in (1):

$$
\nabla \times E \cdot \mathbf{n}+\frac{\partial B}{\partial t} \cdot \mathbf{n}=0
$$

Since the T-A model approximates the superconducting layer as a sheet, the Faraday's law is applied to the sheet geometry. The difference is that a normal vector $\mathbf{n}$ is applied in (1). Then, $E-J$ power law is employed as

$$
E=E_{0}\left(\frac{|J|}{J_{c}}\right)^{n} \frac{J}{J_{c}}
$$

And $J$ is calculated as

$$
J=\nabla \times T
$$

Where, $T$ is current vector potential, $n$ is the n-value of superconducting tape. The magnetic flux density $B$ is solved using the traditional $\mathbf{A}$ formulation, seen in (4). $J c(B)$ is calculated by (5):

$$
\begin{aligned}
& \nabla \times \nabla \times A=\mu_{0} J \\
& J_{c}(B)=J_{c 0}\left(1+\frac{1}{B_{0}} \sqrt{\gamma^{-2} B_{\|}+B_{\perp}}\right)^{-\alpha}
\end{aligned}
$$

$B_{\| /}, B_{\perp}$ are the parallel and perpendicular magnetic filed components; $I_{c 0}$ is the critical current of superconducting tape at $77 \mathrm{~K}$, self-field; $B_{0}=20 \mathrm{mT} ; \gamma=5, \alpha=0.65$ [10]。

The FEM mesh for a $10 \mathrm{~cm}$ length, 3D TSTT composite cable is built by using COMSOL software, seen in Fig.2. The electromagnetic solve equation is replaced by the T-A equation model with applying current to the superconducting tapes by setting the edges. The magnetic flux density and critical current of the demo composite conductor is calculated based on $J_{\mathrm{c}}-B$ relationship. The theoretical critical current is913 A@77 K.

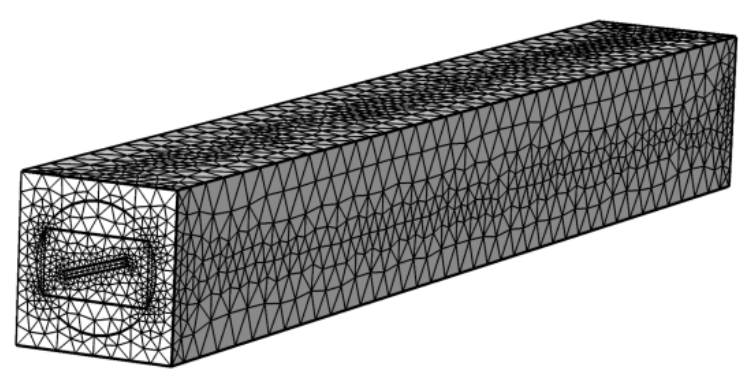

Fig. 2. The mesh of TSTT composite cable based on 3D FEM in COMSOL. 


\section{MANUFACTURE AND CRITICAL CURRENT EXPERIMENTAL TESTS OF THE COMPOSITE CABLE}

\section{A. Continuous HTS Composite Cable Manufacturing}

In this paper, China Electric Power Research Institute (CEPRI) design the manufacturing process of high temperature superconducting internal cooling twisted stacked-tape in tube (TSTT) composite cable using Fujikura REBCO tapes. A continuous TSTT composite cable with 100 meters is manufactured in Anhui Hong Yuan Special Cable Group co. LTD in China. The industrial manufacturing process photo of insulation coating process for the $100 \mathrm{~m}$ composite cable is shown in Fig. 3. Then, a $1.5 \mathrm{~m}$ demo cable is cut off from the $100 \mathrm{~m}$ cable and to be tested for its critical current characteristic after the industrial manufacturing.

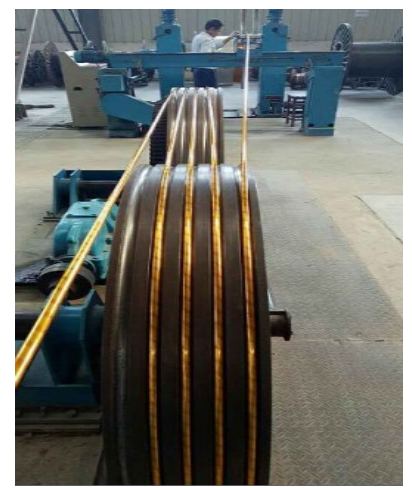

Fig. 3. Photo of $100 \mathrm{~m}$ HTS composite cable in insulation coating process.

\section{B. The critical current testing system for TSTT cable}

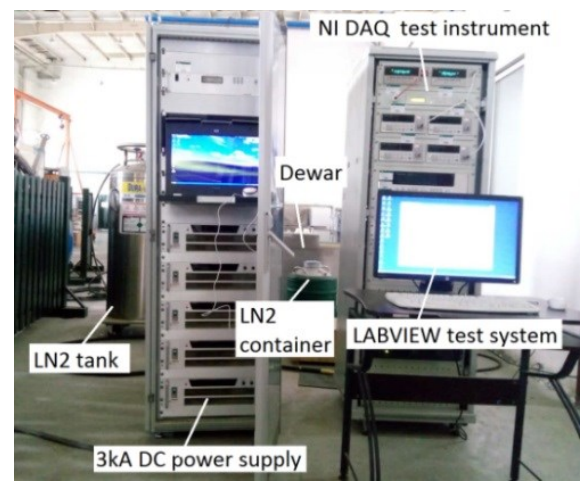

Fig. 4. $V$-I test system photo for composite HTS cable.

The $V$-I test platform photo is shown in Fig. 4. The test system consists of a NI Data Acquisition (DAQ) test instrument, a $3 \mathrm{kA}$ DC power supply with quench protection circuit and a cryogenic dewar. A $1.5 \mathrm{~m}$ demo composite cable is placed in a vessel which is filled with liquid nitrogen at $77 \mathrm{~K}$. Each superconducting tape is connected to the $3 \mathrm{kA}$ DC power supply through a silver current lead in the $V-I$ test system. The cross section of a silver current lead is $10 \mathrm{~mm} \times 0.4 \mathrm{~mm}$. There are several voltage leads both on REBCO tapes and silver current leads. And they are all connected to the DAQ test instrument. All of the current and voltage signals of TSTT composite cable are collected by DAQ system. Therefore, the current sharing characteristics in the composite cable can be obtained according to the voltage signals of the silver leads and their resistance. So, the experimental $V-I$ curve and the quench state of the $1.5 \mathrm{~m}$ demo TSTT composite cable is obtained. In the experiment, the shunt ratio for the total current is $2000 \mathrm{~A} / 75 \mathrm{mV}$, the charging rate for the composite cable is $2 \mathrm{~A} / \mathrm{s}$ and the discharging rate is $5 \mathrm{~A} / \mathrm{s}$. Fig. 5 is the $1.5 \mathrm{~m}$ TSTT composite cable with $160 \mathrm{~mm}$ bending radius.

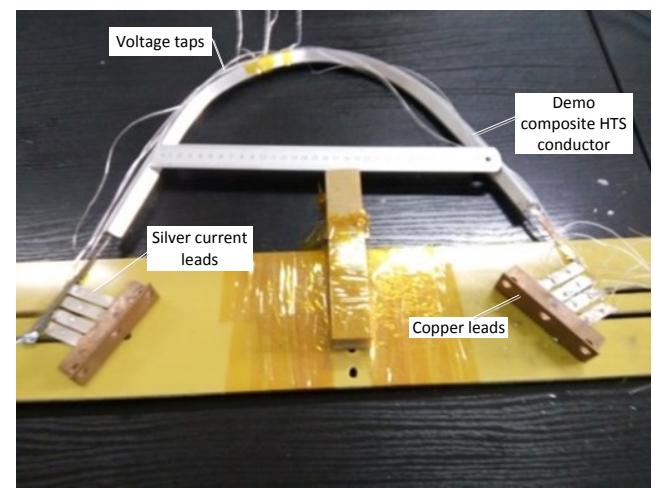

Fig. 5. Photo of silver current lead and welding condition of voltage leads for $1.5 \mathrm{~m}$ TSTT composite cable with $160 \mathrm{~mm}$ bending radius.

\section{Critical current testing for straight composite cable}

Fig. 6 is the $V$ - $I$ curves of four REBCO tapes which are in a straight TSTT composite cable. The critical currents of four HTS tapes are $211 \mathrm{~A}, 215 \mathrm{~A}, 236 \mathrm{~A}$ and $237 \mathrm{~A}$, respectively. The critical current of the $1^{\text {st }}$ tape and $2^{\text {nd }}$ tape are both less than that of the $3^{\text {th }}$ tape and $4^{\text {th }}$ tape. This decrease in the critical current may be due to the damage caused by the composite cables in the manufacturing process, or in the welding process due to the high temperature environment. Compared to the critical current of single REBCO tape with 270 A@77 K, the critical currents of four tapes have decreased by $21.85 \%, 20.37 \%, 12.59 \%$ and $12.22 \%$. The maximum reduction ratio of $1^{\text {st }}$ tape is nearly $22 \%$.

Fig. 7 is the critical current curve for the TSTT composite cable. When one tape takes place quench at the first time, we add the current of each tape at this moment as the overall critical current of the cable. According to the transition criterion $(1 \mu$ $\mathrm{V} / \mathrm{cm}$ ), the critical current is $780 \mathrm{~A}$ and it is $85.43 \%$ of the theoretical value of $913 \mathrm{~A}$.

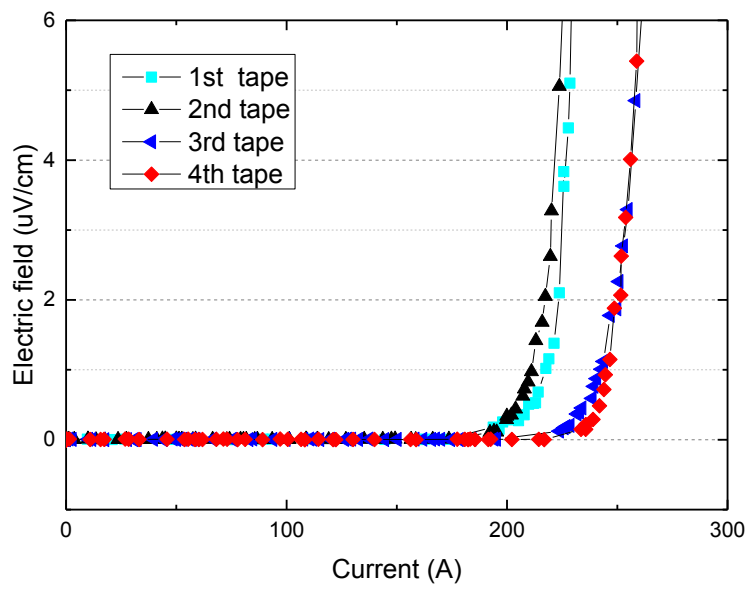

Fig. 6. Experimental $V-I$ curve of each REBCO tape in HTS TSTT composite cable. 


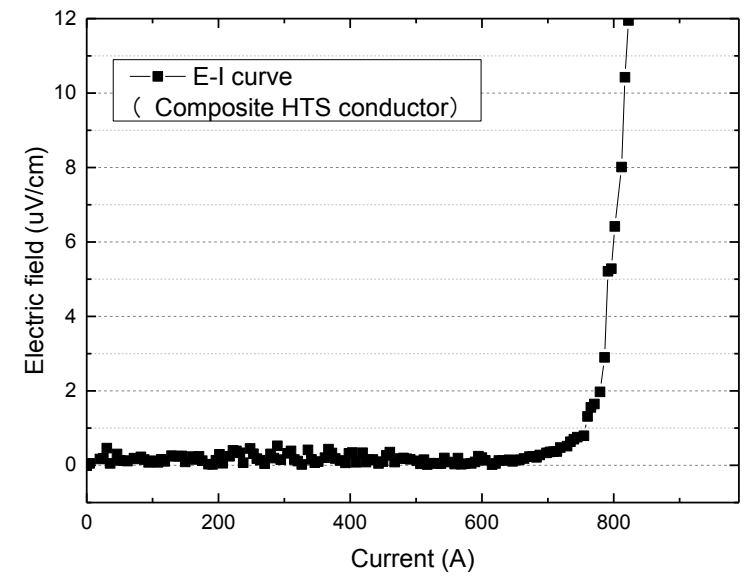

Fig. 7. Experimental $V-I$ characteristic of HTS TSTT composite cable.

\section{Critical current testing for composite cable with bending}

To investigate the bending effect on the critical current in the process of winding, this article has carried on a critical current test of composite cable with the bending radius of $160 \mathrm{~mm}$ which is the inner radius of a pancake for a superconducting energy storage magnet [11].

Fig. 8 is the $V$ - $I$ curves of four REBCO tape in a bending composite cable. Fig. 9 is the $V$ - $I$ curve for the composite cable after bending. In Fig. 8, the critical currents of four HTS tapes are $183 \mathrm{~A}, 185 \mathrm{~A}, 218 \mathrm{~A}$ and $220 \mathrm{~A} @ 77 \mathrm{~K}$. These critical currents have decreased by $32.22 \%, 31.48 \%, 19.25 \%$ and $18.51 \%$ compared to the critical current of a REBCO tape, respectively. The critical currents of the $1^{\text {st }}$ tape and $2^{\text {nd }}$ tape that have been damaged in the process of manufacturing are more attenuated than that of the $3^{\text {th }}$ tape and $4^{\text {th }}$ tape. The critical current of the bending composite cable is $700 \mathrm{~A}$ which is $89.74 \%$ of that of the straight composite cable. It is shown that bending leads to further reduction of the critical current for composite cable.

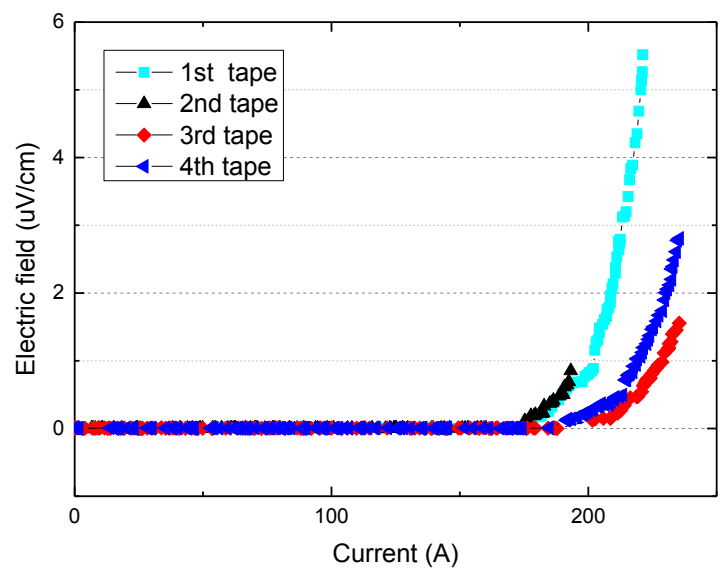

Fig. 8. Experimental $V-I$ curve of each REBCO tape in HTS TSTT composite cable with $160 \mathrm{~mm}$ bending radius.

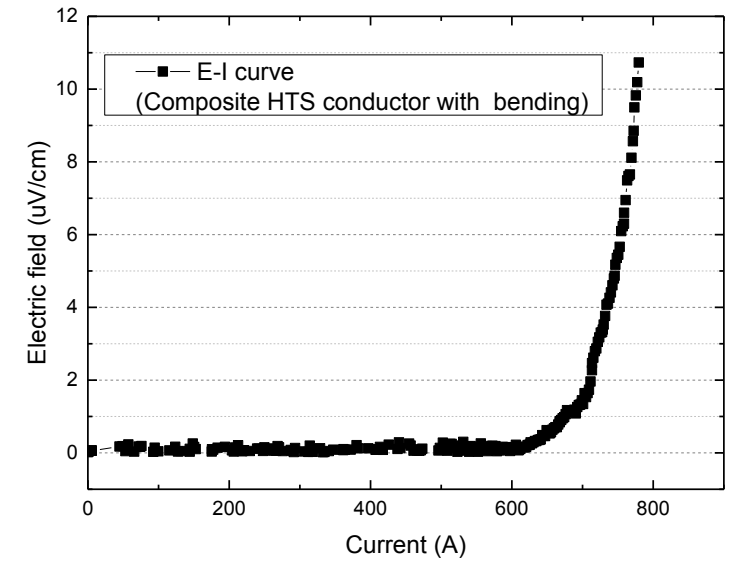

Fig. 9. Experimental $V-I$ characteristic of HTS TSTT composite cable with 160 $\mathrm{mm}$ bending radius.

\section{E. Comparison and analysis}

Considering the influence of magnetic field and mechanical processing, the calculated critical current of the composite cable is $913 \mathrm{~A}$. After manufacturing, the critical current reduces to $780 \mathrm{~A}$ and further to $700 \mathrm{~A}$ with a bending radius of $160 \mathrm{~mm}$. Compared to the theoretical value, the critical current reduction ratio of straight and bending HTS TSTT composite cable are $14.5 \%$ and $23.3 \%$, respectively, as in Table. 2 . When the composite cable is bent, its critical current is nearly $90 \%$ of the straight one. Therefore, the composite cable meets the critical current performance requirements. It is feasible to use this composite cable to make a designed MJ class SMES magnet. We will give these research achievements in future.

TABLE II

THE CRITICAL CURRENT COMPARISON BETWEEN CALCULATION AND EXPERIMENTAL RESULTS OF TSTT COMPOSITE CABLE

\begin{tabular}{cccc}
\hline \hline & \multirow{2}{*}{$\begin{array}{c}\text { Theoretical } \\
\text { value }\end{array}$} & \multicolumn{2}{c}{$\begin{array}{c}\text { Experimental Ic value of composite } \\
\text { HTS conductor }\end{array}$} \\
\cline { 3 - 4 } & 913 & Straight & Bending \\
\hline $\begin{array}{c}\text { Critical current } \\
\text { /A@77K } \\
\begin{array}{c}\text { Reduction ra- } \\
\text { tio }\end{array}\end{array}$ & - & 780 & 700 \\
\hline \hline
\end{tabular}

\section{CONCLUSION}

A high temperature superconducting twisted stacked-tape in tube (TSTT) composite cable is proposed in CEPRI with a spiral cooling tunnel inside and a metal jacket. A T-A equation model is applied to calculate the theoretical critical current of the TSTT composite cable. A continuous TSTT cable with 100 meters is manufactured in China. The critical current experimental system is built for the composite cable. Compared to the theoretical value with913 A@77 K, the critical current reduction ratio of straight and bending TSTT composite cable are $14.5 \%$ and $23.3 \%$. In addition, after the composite cable is bent, its critical current is nearly $90 \%$ of the straight one. Therefore, the composite cable is considered as the potential superconducting material for a MJ SMES magnet. 


\section{REFERENCES}

[1] M. H. Ali., "An overview of SMES applications in power and energy systems," IEEE Trans. Sustainable Energy, vol.1, 2010, pp.38-47.

[2] Nagaya, S., et al. "Development of MJ-class HTS SMES for bridging instantaneous voltage dips," IEEE Trans. Applied Superconductivity, vol.14, no.2, 2004, pp:770 - 773.

[3] M. Park, et al. "Conceptual Design of HTS Magnet for a 5 MJ Class SMES," IEEE Trans. Applied Superconductivity, vol.18, no.2, 2008, pp: $750-753$

[4] M. Sander. "LIQHYSMES - A 48 GJ Toroidal MgB2-SMES for Buffering Minute and Second Fluctuations," IEEE Trans. Applied Superconductivity, vol.23, no.3, 2013, Art.no. 5700505.

[5] M. Takayasu, L. Chiesa, L. Bromberg, and J. V. Minervini, "HTS twisted stacked-tape cable conductor", Superconductor Science and Technology ,no.25, 2012, Art.no.014011.

[6] K. Philipp, V. Zermeno, M. Takayasu and F. Grilli, "Three-Dimensional numerical simulations of twisted stacked tape cables." IEEE Transactions on Applied Superconductivity vol.25, no. 3, 2015, Art.no.4801505.

[7] B. Shen, J. Li, J. Geng, L. Fu, X. Zhang, H. Zhang, C. Li, F. Grilli and T. A. Coombs, "Investigation of AC losses in horizontally parallel HTS tapes", Superconductor Science and Technology, vol. 30, no. 7, 2017.

[8] Grilli, F., Zermeño, V. M., Takayasu, M. "Numerical modeling of twisted stacked tape cables for magnet applications". Physica C: Superconductivity and its Applications, vol.518, 2015, pp: 122-125.

[9] Ming Qiu, Shuangquan Rao, Jiahui Zhu, PanPan Chen, Shanshan Fu, Weijia Yuan, and Jun Gong. "Mechanical Properties of MJ-Class Toroidal Magnet Wound by Composite HTS Conductor". IEEE Transactions on Applied Superconductivity, vol.27, no.4, 2017, Art.no. 5700605.

[10] H. Zhang. "An efficient 3D finite element method model based on the TA formulation for superconducting coated conductors," Supercond. Sci. Technol.no.2, 2017, Art.no.024005.

[11] M. Qiu. "Energy Storage Characteristics of MJ-Class Toroidal HTSSMES Considering Maximum Value of Perpendicular Magnetic Field," The 8th International Conference on Applied Energy - ICAE2016, October 8-11, 2016, Beijing, China. 